\title{
Spontaneous Scleral Perforation of an Anterior Chamber Intraocular Lens
}

\author{
Oriel Spierer ${ }^{a, b} \quad$ Terrence P. O'Brien ${ }^{b}$ \\ ${ }^{a}$ Ophthalmology Division, Tel Aviv Sourasky Medical Center, Sackler Faculty of Medicine, \\ Tel Aviv University, Tel Aviv, Israel; ${ }^{b}$ Bascom Palmer Eye Institute, University of Miami \\ Miller School of Medicine, Palm Beach Gardens, Fla., USA
}

\section{Keywords}

Scleral perforation · Cataract surgery · Anterior chamber intraocular lens · Anterior segment optical coherence tomography · White-to-white diameter

\begin{abstract}
A routine eye examination of a 69-year-old man revealed a scleral perforation of one of the haptics of the anterior chamber intraocular lens (AC IOL) which had been implanted many years ago. The patient was asymptomatic with good visual acuity. His history was negative for any trauma, eye rubbing, topical corticosteroid use, or autoimmune disease. The horizontal and vertical white-to-white diameters of the cornea in the right eye were 11.5 and 10.5 $\mathrm{mm}$, respectively. Anterior segment optical coherence tomography showed the anterior chamber length to be $12.28 \mathrm{~mm}$ horizontally and $10.63 \mathrm{~mm}$ vertically. The patient underwent an IOL exchange, and the length of the explanted AC IOL was measured to be $12 \mathrm{~mm}$. We speculate that the AC IOL, which was vertically aligned, was oversized. This case demonstrates the need for proper sizing and positioning of an AC IOL. In complex cases where AC IOL may be used, measuring the horizontal and vertical lengths of the anterior chamber by anterior segment optical coherence tomography prior to surgery may be useful.
\end{abstract}




\section{Introduction}

The use of an anterior chamber intraocular lens (AC IOL) in cataract surgery is uncommon these days, due to improvement in surgical techniques and IOL design and materials. The most common complications of AC IOL are corneal decompensation and persistent inflammation [1]. These as well as other complications may necessitate IOL explantation. AC IOL expulsion or exposure is rare [2], and usually the result of trauma, often after accidental falling [3].

We report the case of an asymptomatic patient with spontaneous scleral perforation of a vertically aligned AC IOL which, we speculate, was oversized.

\section{Case Report}

A 69-year-old man was referred to our institute because of subconjunctival dislocation of one of the AC IOL haptics, which was found during a routine eye examination in another clinic. Examination of the right eye revealed a best corrected visual acuity of 20/25 and intraocular pressure of $11 \mathrm{~mm} \mathrm{Hg}$. A vertically aligned open-loop PMMA IOL was seen in the anterior chamber. The conjunctiva was white and quiet, but one of the AC IOL haptics was found to perforate the superior sclera under the intact conjunctiva. The cornea was clear and the anterior chamber was in normal depth with no inflammatory signs (fig. 1). The fundus of the right eye was normal, and an examination of the left eye was unremarkable. The AC IOL had been implanted many years ago during a cataract surgery. The patient denied any decreased vision, pain, or discomfort in his right eye. Due to the risk of conjunctival erosion, infection, and endophthalmitis, the AC IOL was explanted, a sclera-fixated posterior chamber IOL was implanted, and the scleral wound was repaired. The overall length of the explanted AC IOL was measured to be $12 \mathrm{~mm}$. The horizontal and vertical white-to-white (WTW) diameters of the cornea of the right eye were 11.5 and $10.5 \mathrm{~mm}$, respectively. Anterior segment optical coherence tomography (AS OCT; Visante; Carl Zeiss Meditec Inc., Dublin, Calif., USA) showed the anterior chamber length to be $12.28 \mathrm{~mm}$ horizontally and $10.63 \mathrm{~mm}$ vertically (fig. 2).

\section{Discussion}

Significant improvement in cataract surgery and IOL design during the last decades resulted in low rates of AC IOL implantation. Yet, in cases where posterior chamber IOL cannot be safely implanted, other options should be considered. Scleral or iris posterior chamber IOL fixation may be the preferred option; however, not every cataract surgeon is experienced in IOL suturing, and in this case AC IOL is an acceptable alternative. Nevertheless, AC IOL are associated with relatively high rates of complications including pseudophakic bullous keratopathy, anterior uveitis, erosion through the iris, pupillary block glaucoma, and cystoid macular edema $[1,4-6]$. Subconjunctival dislocation of the lens into the sclera is a rare complication of an AC IOL, which necessitates lens explantation and wound repair to prevent endophthalmitis [7]. Gungel et al. [7] reported the case of a woman with a history of allergy and pruritus in both eyes who presented with bilateral exposure of the AC IOL haptic tips through the limbus as well as with unilateral endophthalmitis. Vigorous rubbing of the eye had most probably caused limbal erosion that had led to perforation of the AC IOL haptic 
through the limbus [7]. In the current case, the patient's ocular history was negative for any trauma or eye rubbing. The patient also did not have a history of autoimmune disease. The possibility that long-term use of topical corticosteroids may result in scleral thinning in a healthy eye is controversial $[8,9]$; however, in our case the patient denied the use of topical corticosteroids, and on clinical examination there were no areas of scleral thinning.

Accurate AC IOL sizing is crucial, as oversized AC IOL can cause peripheral anterior synechiae [6], corneal astigmatism [10], and very rarely perforation through the sclera, as suspected in the present case. External WTW diameter measurements may be used as an estimation of the internal diameter of the anterior chamber. A common practice for selecting the appropriate size of an AC IOL is to add $1 \mathrm{~mm}$ to the horizontal WTW measurement. WTW distances differ with each measuring tool (i.e. caliper IOL Master, Carl Zeiss Meditec Inc.; Orbscan: Bausch + Lomb, Garden City, N.Y., USA), and these tools seem to be crude, only allowing estimation of the internal anterior chamber diameter. Therefore, AS OCT, which can directly measure the internal anterior chamber diameter, is preferred [11]. In the present case, the WTW and Visante AS OCT measurements indicated clearly that the 12-mm AC IOL was too large to be vertically aligned in the anterior chamber. We hypothesize that this was a risk factor for the spontaneous scleral perforation. As the cataract surgery had been done many years ago, it is unknown whether the AC IOL had been placed vertically by the surgeon or whether it had been placed horizontally and had rotated later.

Our case demonstrates the need for proper sizing and positioning of an AC IOL, since it might result in spontaneous scleral perforation many years after cataract surgery. This can be done during surgery with the aid of WTW measurement. However, when preparing for complex cataract surgeries in which an AC IOL is expected to be necessary, such as in pseudoexfoliation syndrome or zonular instability, measuring the length of the anterior chamber using AS OCT prior to surgery may be useful.

\section{Statement of Ethics}

The authors have no ethical conflicts to disclose.

\section{Disclosure Statement}

None of the authors have any conflict of interest in regard to the material in this case report.

\section{References}

-1 Auffarth GU, Wesendahl TA, Brown SJ, Apple DJ: Are there acceptable anterior chamber intraocular lenses for clinical use in the 1990s? An analysis of 4,104 explanted anterior chamber intraocular lenses. Ophthalmology 1994;101:1913-1922.

-2 Kumar A, Nainiwal SK, Dada T, Ray M: Subconjunctival dislocation of an anterior chamber intraocular lens. Ophthalmic Surg Lasers 2002;33:319-320.

3 Assia EI, Blotnick CA, Powers TP, Legler UF, Apple DJ: Clinicopathologic study of ocular trauma in eyes with intraocular lenses. Am J Ophthalmol 1994;117:30-36.

Moses L: Complications of rigid anterior chamber implants. Ophthalmology 1984;91:819-825. Seymour RG, Ramsey MS: Cause of intraocular lens removal. Can J Ophthalmol 1989;24:152-154. Smith SG, Holland E, Peterson J: Peripheral anterior synechiae formation with anterior chamber intraocular lenses. Ophthalmic Surg 1992;23:316-319. 
Spierer and O'Brien: Spontaneous Scleral Perforation of an Anterior Chamber Intraocular Lens

7 Gungel H, Altan C, Baylancicek DO: Endophthalmitis due to exposure of anterior chamber intraocular lens haptic tip. J Cataract Refract Surg 2009;35:1633-1636.

-8 Kumar V, Nicholson L, Shankar J, Goel N: Possible causes for exposure of anterior chamber intraocular lens haptic tip. J Cataract Refract Sur 2010;36:703.

-9 Mackool RJ: Corneal/scleral thinning and corticosteroids. J Cataract Refract Surg 2010;36:2018.

-10 Abdel-Hakim AS: Corneal astigmatism induced by oversized rigid anterior chamber implants. J Am Intraocul Implant Soc 1985;11:474-479.

11 Kim SK, Kim HM, Song JS: Comparison of internal anterior chamber diameter imaging modalities: 35 MHz ultrasound biomicroscopy, Visante optical coherence tomography, and Pentacam. J Refract Surg 2010;26:120-126.
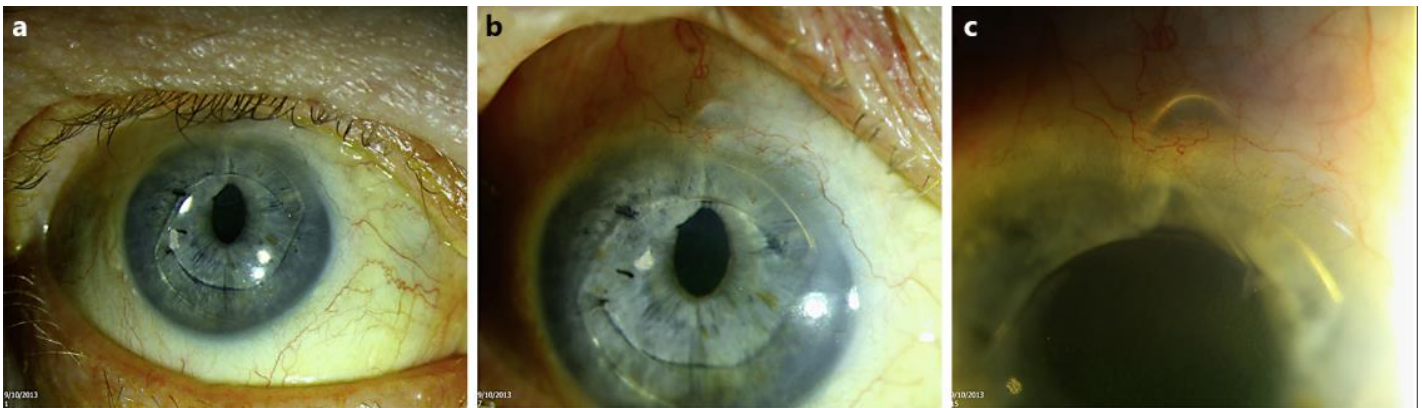

Fig. 1. Slit-lamp photographs of the right eye. a The AC IOL is vertically aligned in the anterior chamber. b, c After lifting the upper eyelid, one of the haptics was found to be perforating the superior sclera and placed under the intact conjunctiva.

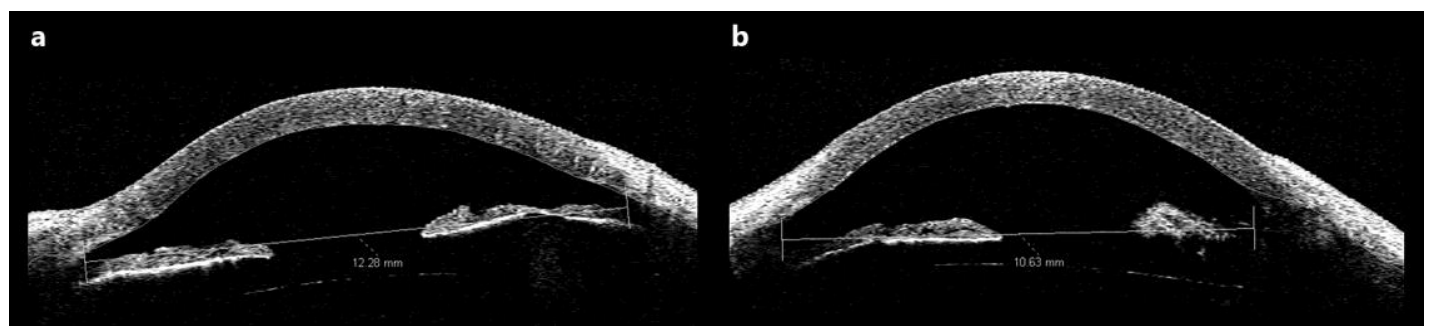

Fig. 2. AS OCT of the right eye. The horizontal anterior chamber length was $12.28 \mathrm{~mm}$ (a). The vertical anterior chamber length was $10.63 \mathrm{~mm}$ (b). 